\title{
Human RAB24, interestingly and predominantly distributed in the nuclei of COS-7 cells, is colocalized with cyclophilin A and GABARAP
}

\author{
MAOQING WU*, GANG YIN*, XIN ZHAO, CHAONENG JI, SHAOHUA GU, \\ RONG TANG, HAI DONG, YI XIE and YUMIN MAO \\ State Key Laboratory of Genetic Engineering, Institute of Genetics, School of Life Science, \\ Fudan University, Shanghai 200433, P.R. China
}

Received July 15, 2005; Accepted August 30, 2005

\begin{abstract}
As an atypical member of the Rab family, Rab24 has several attributes distinguishing this protein from the other members. Based on the yeast two-hybrid system, interaction between human RAB24 and two proteins, cyclophilin A (CyP-A) and gamma-aminobutyric acid type A receptorassociated protein (GABARAP), was detected and identified in COS-7 cells. Interestingly, RAB24 is predominantly localized in the nuclei of COS-7 cells, which is different from previous reports using other cell lines. RAB24 (D123I) can trigger the accumulation of intracellular inclusions, with small quantities of intranuclear inclusions in some cells. The GTPase activity of RAB24 and its two mutants was detected.
\end{abstract}

\section{Introduction}

Rab24 is an atypical Rab protein whose function is currently unknown. Subcellular localization experiments showed that Rab24 is distributed in the endoplasmic reticulum/cis-Golgi region and on late endosomal structures, and this subcellular distribution pattern of Rab24 may indicate its involvement in autophagy-related processes $(1,2)$. Rab24 presents unusual characteristics, including low intrinsic GTPase activity, and is inefficiently prenylated when compared with other Rab proteins (3). Another study also suggested that Rab24 might play a unique role in the degradation of misfolded cellular proteins or trafficking of proteins to the nuclear envelope (4). It has been reported that GFP-Rab24 is associated with

Correspondence to: Dr Yumin Mao, State Key Laboratory of Genetic Engineering, Institute of Genetics, School of Life Science, Fudan University, Shanghai 200433, P.R. China

E-mail: ymmao@fudan.edu.cn

*Contributed equally

Key words: RAB24, cyclophilin A, gamma-aminobutyric acid type A receptor-associated protein, subcellular distribution, colocalization, GTPase activity autophagosomes in amino-acid starved cells; furthermore, Rab24 could be colocalized with LC3 (microbule-associated protein light chain 3, a mammalian homolog of the yeast protein Apg8/Aut7), which suggested that Rab24 protein may be involved in the autophagic pathway since LC3 is an essential gene for autophagy (2). Although a possible role of Rab24 in autophagy has been suggested, its specific physiological function in mammalian cells remains to be defined. It should be mentioned that all of these study results were based on Rab24 from mice. For human RAB24, which varies by two amino acids (localized at the nonconservative region) and shares $98 \%$ identity with Rab24, there is no functional study, although it has been identified and characterized (5).

Based on the yeast two-hybrid system, three proteins interacting with RAB24, human GABARAP [ $\gamma$-aminobutyricacid-type-A (GABAA)-receptor-associated protein], cyclophilin A (CyP-A) and TUBA6 (human tubulin alpha 6), were detected, and the former two were individually cotransfected with RAB24 into COS-7 cells for further identification. The findings on the association between RAB24 and GABARAP and CyP-A in mammalian cells are helpful for further functional study on these three proteins.

It was surprising to find that human RAB24 is localized predominantly in the nuclei of COS-7 cells, which is different from previous reports that Rab24 is only distributed in the cytoplasm of other cell lines. RAB24 has low GTPase activity, and two mutants of RAB24 [RAB24 (Y17A) and RAB24 (S67Q)] produced a substantial increase in RAB24 GTPase activity, which has been suggested previously using Rab24 from mice.

\section{Materials and methods}

Strains, media, plasmids and cell line. The E. coli strains DH5a and BL21 (Rosseta) were grown in Luria Broth medium in the presence of antibiotics, as required for different plasmids. pLexA, pEGFP-C1 and pDsRed-N1 plasmids were purchased from Clontech, and pGEX-4T1 was from Amersham Pharmacia. COS-7 (African green monkey kidney cells) was purchased from the Chinese Academy of Science, cultured and stored in our laboratory. Media and reagents for cell culture were purchased from Invitrogen. 

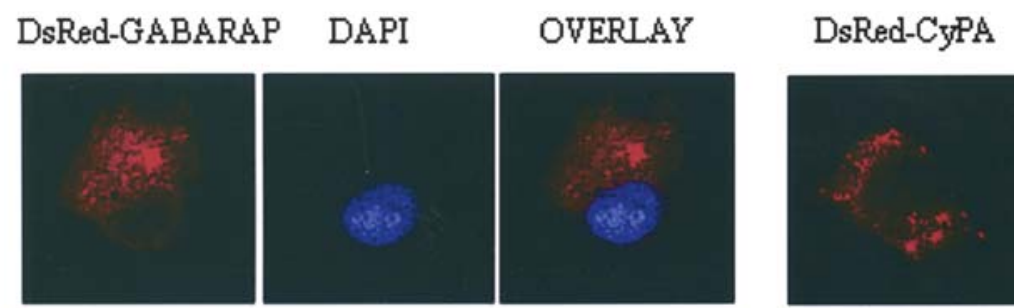

DAPI

OVERLAY
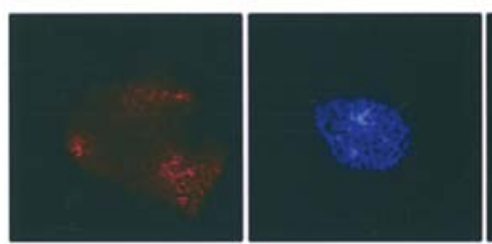

A
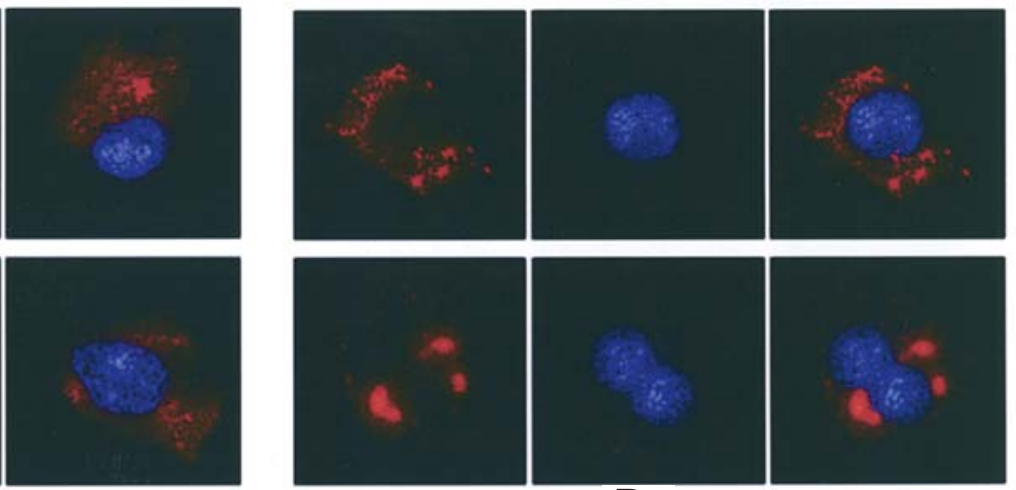

B

Figure 1. Subcellular distribution of human GABARAP and CyP-A in cultured COS-7 cells. The $2 \mu \mathrm{g}$ constructs of pDsRed-N1/CyP-A or pDsRedN1/GABARAP were transfected individually into COS-7 with $3 \mu 1$ Lipofectamine 2000. The nucleus was counterstained with DAPI and viewed using an R2100 Confocal Microscope with the appropriate filters $(543 \mathrm{~nm}$ ). (A) Subcellular localization of DsRed-GABARAP, predominantly distributed in the cytoplasm. (B) Subcellular localization of DsRed-CyP-A, distributed only in the cytoplasm, often encircling the nucleus.

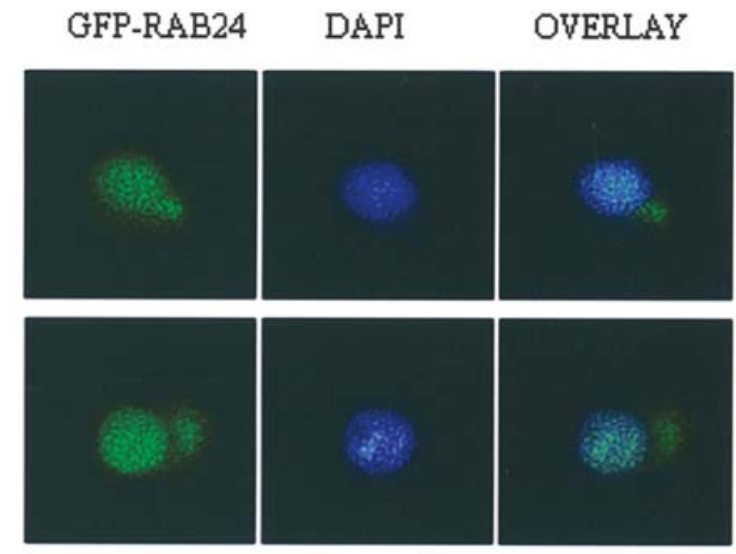

A

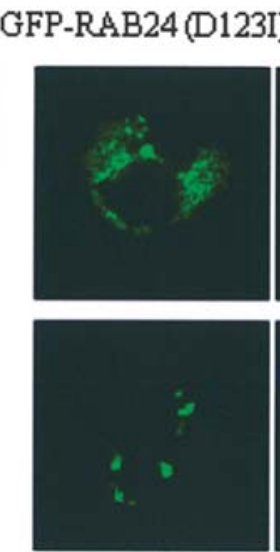

DAPI
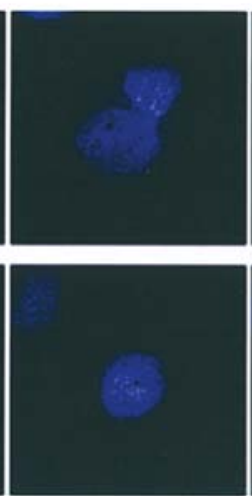

B
OVERLAY
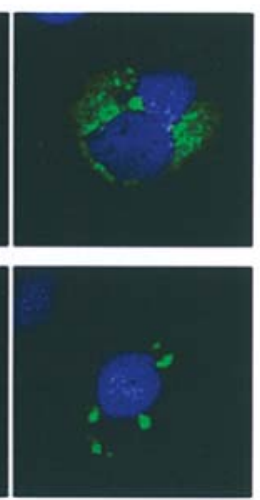

Figure 2. Subcellular distribution of human RAB24 and RAB24 (D123I) in cultured COS-7 cells. The $2 \mu \mathrm{g}$ constructs of pEGFP-C1/ RAB24 or pEGFP-C1/ RAB24 (D123I) were transfected individually into COS-7. The samples were viewed using an R2100 Confocal Microscope with appropriate filters (488 nm). (A) Subcellular localization of GFP-RAB24, predominantly distributed in the nucleus, with small amounts at the perinuclear position. The distribution pattern would be quite different with other cell lines. (B) Subcellular localization of GFP-RAB24 (D123I) collectively form intracellular inclusions, mostly encircling the nucleus (possibly in the endoplasmic reticulum) and without the ability to gain passage. In some cells, intranuclear inclusions were visible, but the nuclear envelope is disrupted (two lower panels).

Expression constructs. The cDNA encoding RAB24 was obtained by PCR amplification of the recombinant clone constructed by our laboratory and cloned into various plasmids, including pLexA, pEGFP-C1 and pGEX-4T1. The RAB24 cDNA was altered by overlap-extension PCR, using the appropriate mutator oligonucleotides (listed below) to introduce amino acid substitutions at D123 (D123I, pEGFPC1 and pGEX-4T1), Y17 (Y17A, pGEX-4T1), and S67 (S67Q, pGEX-4T1). ORFs of CyP-A and GABARAP were obtained using PCR amplification for human multiple tissue cDNA, and cloned into plasmid pDsRed-N1, respectively. All primers and restriction enzymes of expression constructs are: pLexA-RAB24 sense (EcoRI) 5'-CGGAATTCGCGA AGACGTACGATTATCTCTTC-3', and antisense (XhoI) 5'CCGCTCGAGAAGTAGCGAGCAACGAAAGAAACTG3'; pEGFP-C1-RAB24 sense (EcoRI) 5'-CGGAATTCTAGC GGGCAGCGCGTGGACGTCAA-3', and antisense (BamHI) 5'-CGGGATCCGTGATGACAACAGCTGTAGAAGTAG G-3'; pGEX4T1-RAB24 sense (BamHI) 5'-CGGGATCCA
GCG GGCAGCGCGTGGACGTCAA-3', and antisense (XhoI) 5'-CCGCTCGAGGTGATGACAACAGCTGTAGAAGTA GG-3'; RAB24 (Y17A) 5'-CGGGATCCAGCGGGCAGC GCGTGGACGTCAAGGTGGTGATGCTGGGCAAGGCC AAGAGTATCCTGCTGGAAGAAGACCGGAGG-3'; pDsRed-N1-CyP A sense (HindIII) 5'-CCAAGCTTACCA TGGAGTTCGTGTACAAAGAAGAGC-3', and antisense (BamHI) 5'-CGGGATCCCGCAGACCGTAGACACTTT CGTAC-3'; pDsRed-N1-GABARAP sense (EcoRI) 5'-CGG AATTCTGACCATGGTCAACCCCACCGTGTTCTTC-3', and antisense (BamHI) CCAAGAGTATCCTGCTGGAAG AAGACCGGAGG-3'; pDsRed-N1-CyP A sense (HindIII) 5'-CCAAGCTTACCATGGAGTTCGTGTACAAAG AAGAGC-3', and antisense (BamHI) 5'-CGGGATCCC GCAGACCGTAGACACTTTCGTCAC-3'; pDsRed-N1GABARAP sense (EcoRI) 5'-CGGAATTCTGACCAT GGTCAACCCCACCGTGTTCTTC-3' and antisense (BamHI) 5'-CGGGATCCCGTTCGAGTTGTCCACAGT CAGCAATG-3'. 


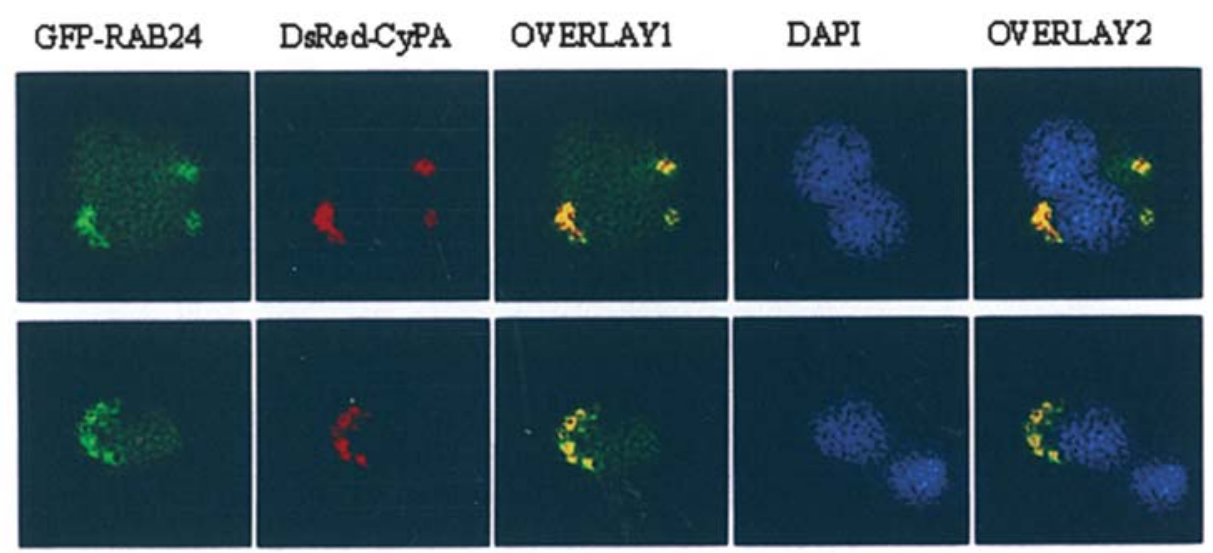

B
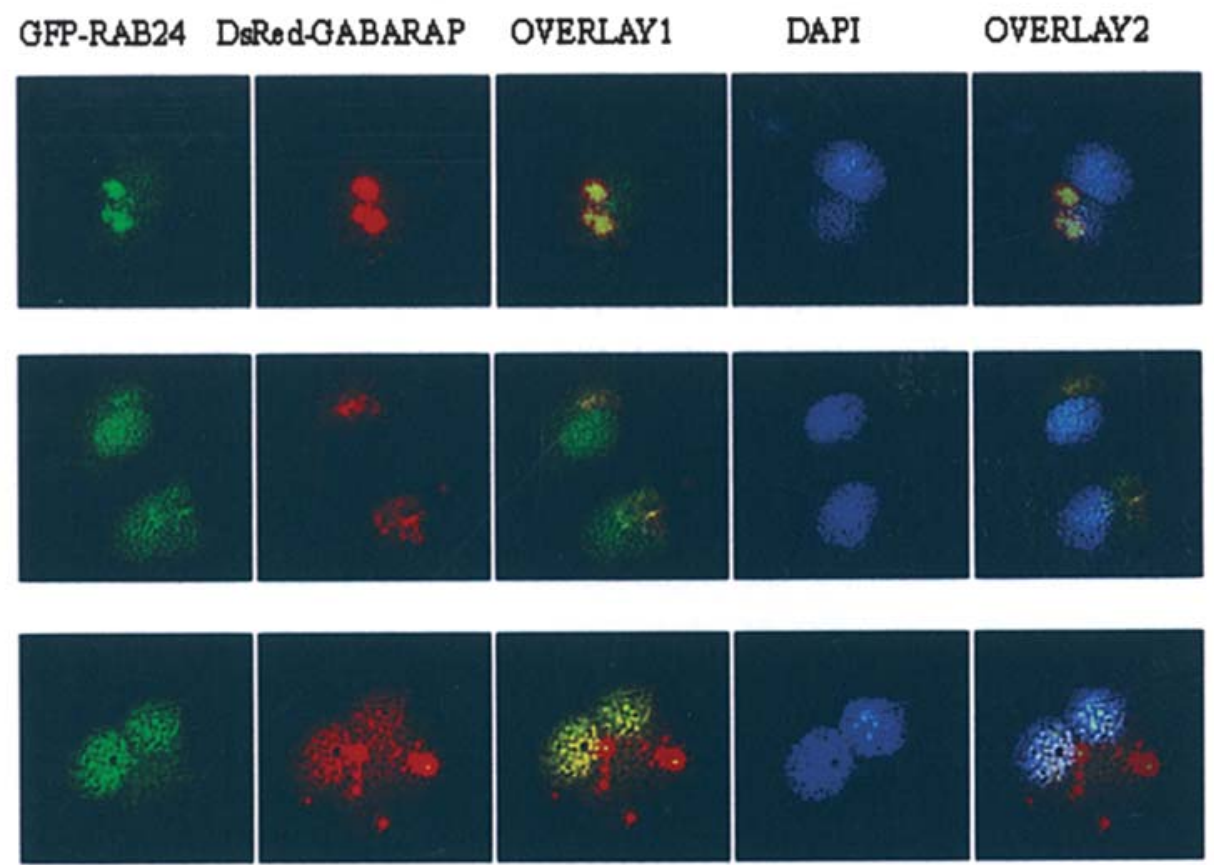

Figure 3. Colocalization of RAB24 and CYP-A or RABARAP in COS-7 cells. The $2 \mu \mathrm{g}$ constructs of pDsRed-N1/CyP-A or pDsRed-N1/GABARAP and $2 \mu \mathrm{g}$ pEGFP-C1/RAB24 were co-transfected individually into COS-7 cells. (A) Colocalization of RAB24 and CyP-A is always distributed around the nucleus in large dots. GFP-RAB24 is also distributed in the nucleus. (B) Colocalization of RAB24 and RABARAP was often distributed in the cytoplasm near the nucleus (two upper panels). Surprisingly, DsRed-GABARAP entered the nucleus and was colocalized with GFP-RAB24 in some cells (low panel).

Cell culture and liposome-mediated transfection. COS-7 cells were grown in Dulbecco's modified Eagle medium contaning $10 \%$ FBS and maintained at $37^{\circ} \mathrm{C}$ in a $5 \% \mathrm{CO}_{2}$ atmosphere. On the day before transfection, cells were plated on coverslips in the 6 -well tissue culture plates. Cells were transfected at 60-80\% confluence with the indicated expression vectors using Lipofectamine 2000 (Invitrogen) according the manufacturer's instructions. Following 32-48 $\mathrm{h}$ transfection, cells were washed 3 times with $1 \mathrm{X}$ PBS, fixed with $4 \%$ paraformaldehyde for 15 min washed 3 times with 1X PBS, counterstained with 4',6'-diamidino-2-phenylindole for $10 \mathrm{~min}$, and washed 2 times with $\mathrm{ddH}_{2} \mathrm{O}$. The samples were then viewed using an R2100 Confocal Microscope (Bio-Rad) with the appropriate filters, and the photos were captured with Leica DC viewer software. The overlays were processed with Photoshop 6.0.

Yeast two-hybrid system. The MATCHMAKER LexA twohybrid system and human fetal brain MATCHMAKER LexA cDNA libraries were purchased from Clontech. The yeast two-hybridization was performed according to the manual supplied by the manufacturer, and a sequential transformation method was adopted. All positive clones were classified by yeast clone hybridization and the length of PCR product using the AD fusion site-specific primer (sense, 5'-CCA GCCTCTTGCTGAGTGGAGATG-3'; antisense, 5'-CGT AAATTTCTGGCAAGGTAGACAAG-3'). The independent clones were further verified with a yeast mating test. PCR products amplified from positive clones were sequenced on an ABI PRISM ${ }^{\mathrm{TM}} 377$ DNA sequencer (Perking-Elmer), and the sequencing results were directly submitted to the NCBI website (http://www.ncbi.nlm.nih.gov/blast) to perform BLAST analysis.

Identification for the interacting proteins of RAB24

Yeast mating test. The yeast mating test was used to verify the interaction between RAB24 and CyP-A, GABARAP and TUBA6. All yeast-mating procedures were performed according to the recommendations of the manufacturer (Clontech). These results are not shown. 
A

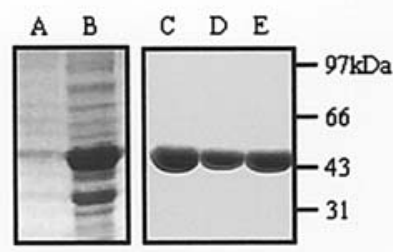

C

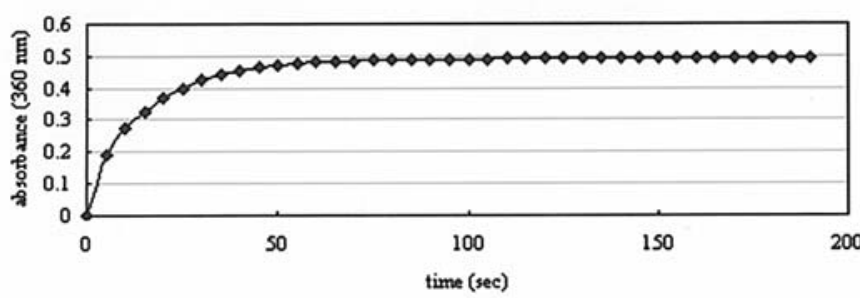

B

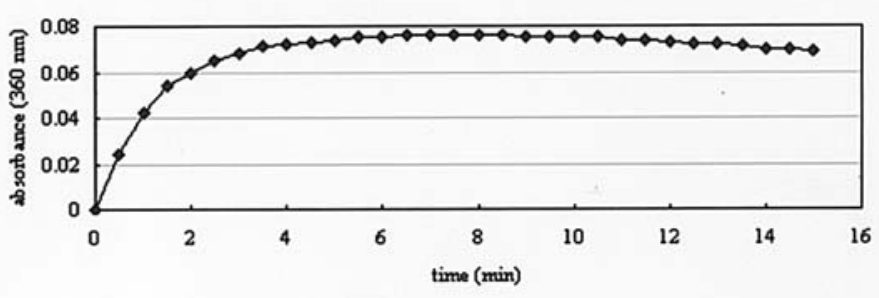

D

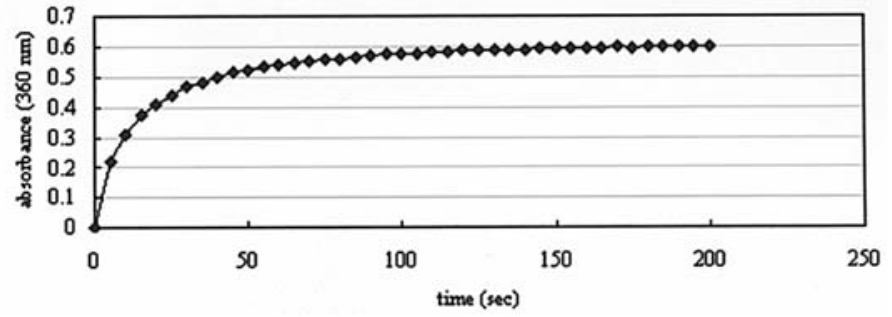

Figure 4. Expression of GST-RAB24 (and its three mutants) and the continuous spectroscopic assay of Pi release from the RAB24-GTP (RAB24 (Y17A)GTP, RAB24 (S67Q)-GTP) complex measured by absorbance at $360 \mathrm{~nm}$ with the MESG/PNP assay system. (A) Expression of GST-RAB24 and its three mutants in E. coli. (A) Supernatant of RAB24 (D123I); (B) pellet of RAB24 (D123I); (C) supernatant of RAB24 (Y17A); (D) supernatant of RAB24 (S67Q); and (E) supernatant of RAB24. Unlike RAB24 and two other mutants, RAB24 (D123I) shifts to form nearly complete inclusions. (B) Low GTPase activity of RAB24 was detected, with an absorbance below 0.08. (C and D) GTPase activity of RAB24 (Y17A) and RAB24 (S67Q) dramatically increased when compared to that of RAB24. 'S67' appears to have a more negative effect than 'Y17A' on the GTPase activity of RAB24 since RAB24 (S67Q) has a higher absorbance $(0.60)$ than that $(0.50)$ of RAB24 (Y17A) under the same conditions.

Colocalization of the interacting proteins and RAB24 in vivo. The $2 \mu \mathrm{g}$ constructs of pDs Red-N1/CyP-A or pDs RedN1/GABARAP and $2 \mu \mathrm{g}$ pEGFP/RAB24 were mixed in $250 \mu \mathrm{l}$ DMEM (no serum), and $5 \mu 1$ Lipofectamine 2000 was also mixed gently in $250 \mu \mathrm{l}$ DMEM (no serum). After 5 min, they were mixed together for $20 \mathrm{~min}$. These mixtures were then co-transfected individually into COS-7 cells and cultured on coverslips in 6-well plates.

GTPase activity detection for human RAB24 and its mutants. Protein expression and purification. E. coli strain BL21 (Rosseta) containing the expression plasmids was grown overnight in LB medium containing $100 \mathrm{mg} / \mathrm{l}$ ampicillin. Expression of GST-tagged recombinant protein was induced at $\mathrm{OD}_{560}$ of $0.4-0.6$ with $1 \mathrm{mM}$ IPTG for $4 \mathrm{~h}$ at $24^{\circ} \mathrm{C}$, then the cells were harvested by centrifugation at $5000 \mathrm{rpm}$ for $15 \mathrm{~min}$ at $4^{\circ} \mathrm{C}$. The cells were resuspended in $1 \mathrm{X}$ PBS with $1 \mathrm{mM}$ PMSF, then lysed by sonication for $10 \mathrm{~min}$ in an ice bath. The lysate was cleared by centrifugation at $14000 \mathrm{rpm}$ for $15 \mathrm{~min}$ at $4^{\circ} \mathrm{C}$. After centrifugation, the supernatant was loaded to a glutathione sepharose $4 \mathrm{~B}$ column. GST fusion proteins were eluted and analyzed by SDS-PAGE.

GTPase activity assay for RAB24 and its mutants. An EnzChek Phosphate Assay kit was purchased from Molecular Probes and utilized according to the manufacturer's protocol. The reaction components are: $50 \mu 120 \mathrm{X}$ reaction buffer, $200 \mu 1 \mathrm{MESG}$ substrate solution, $10 \mu 1 \mathrm{PNP}$ (1U), $200 \mu \mathrm{M}$ GTP, and $5 \mu \mathrm{M}$ GST-RAB24/GST-RAB24 (Y17A)/GST$\mathrm{RAB} 24$ (S67Q), adding $\mathrm{ddH}_{2} \mathrm{O}$ to $1 \mathrm{ml}$ (total volume).

\section{Results}

Subcellular distribution of RABARAP, CyP-A, RAB24 and RAB24 (D123I) in COS-7. RABARAP and CyP-A are all localized in the cytoplasm of COS-7 cells (Fig. 1). The former also appears to be distributed throughout the cytoplasm, with small amounts in the nucleus; but the latter appears to show a perinuclear reticular localization that often encircles the nucleus (Fig. 1B), which is similar to the distribution pattern of RAB24 (D123I) (Fig. 2B). It is no surprise that subcellular distribution of these two proteins is consistent with previous study results obtained by using other cell lines of human and mouse origin.

As shown in Fig. 2A, RAB24 is predominantly localized in the nucleus except for small amounts distributed in the cytoplasm near the nucleus. However, the mutant RAB24 (D123I) accumulated intracellular inclusions that were always targeted to the perinuclear reticulum in most COS-7 cells (Fig. 2B).

Colocalization of RAB24 and RABARAP or CyP-A in COS-7 cells. RAB24 is well colocalized with CyP-A in a perinuclear position (Fig. 3A). For another interacting protein, GABARAP, there are two kinds of subcellular colocalization with RAB24 (Fig. 3B). In some COS-7 cells, RAB24 and GABARAP can colocalize in the cytoplasm (Fig. 3B, upper two panels); in others, however, their colocalization can also be seen in the nucleus (Fig. 3B, lower panel).

GTPase activity detection of RAB24 and its mutants. Consistent with previous reports, the GTPase activity of 
human RAB24 is low (Fig. 4B). The amino acid substitution at two different positions (Y17A and S67Q) resulted in a dramatic increase of GTPase activity of RAB24 (Fig. 4C and D). However, there are no results as yet on GTPase activity of the mutant RAB24 (D123I), which formed inclusion bodies when expressed in E. coli (Fig. 4A).

\section{Discussion}

It has been recognized that Rab24 is distributed in the cytoplasm, especially in the endoplasmic reticulum/cis-Golgi region and on late endosomal structures, with cell lines of BHK, HeLa, MDCK, NmuLi, CHO, HEK 293 and NIH 3T3 mouse fibroblasts. Our study showed an unexpected and surprising result that RAB24 is predominantly localized in the nucleus except for small amounts distributed in the cytoplasm near the nucleus (Fig. 2A). It was reported that HIV could use various elements of endocytic/trafficking machinery mediated by Rab proteins to enter and successfully infect the host cells (6). It is known that RAB24 contains a unique arginine-rich insert, 126EEDRRRRR133 (3), whose latter sequence is reminiscent of unusual arginine-rich nuclear localization signals that allow proteins like HIV tet and rev to undergo nuclear translocation by association with importin- $\beta$, in the absence of importin- $\alpha(7,8)$. Whether or not this arginine-rich sequence serves as a nuclear localization signal for RAB24 in COS-7 cells remains to be confirmed, but we are convinced that this discrepancy of two distribution patterns suggested RAB24 must play a different role in human and monkey cells.

It is of interest that in most COS-7 cells the mutant RAB24 (D123I) accumulated intracellular inclusions that always target the perinuclear reticulum (Fig. 2B). However, in other cell lines (HEK 293 and NIH 3 T3 mouse fibroblasts), it has been reported that the mutant Rab24 GTPase accumulated in nuclear inclusions that disrupted the integrity of the nuclear envelope and appeared to sequester ubiquitin, Hsp70, importin- $\beta$ and GFP-coupled glucocorticoid receptor, which is not found in other Rab GTPases with a similar mutation in the N(T)KXD motif. Based on these results, it has been concluded that Rab24 may play a unique role in the degradation of misfolded cellular proteins or trafficking of proteins to the nuclear envelope if this mutant of Rab24 functions as a dominant suppressor (4). To date, there is no published study that specifically links Rab24 mutations with human disease. However, there are many case reports describing progressive neurodegenerative disorders of unknown etiology, collectively known as neuronal intranuclear inclusion disease (9). According to the possible function of Rab24 mentioned above and our study results, it appears noteworthy that RAB24 may be involved in the degradation of misfolded cellular proteins in the endoplasmic reticulum, where protein folding and degradation occur efficiently (10).

CyP-A is a member of the cyclophilin family, whose functions include protein folding, transport and signaling (11). These immunophilins participate in axonal transport and synaptic vesicle assembly, and may play a role in neuroprotection against abnormal protein aggregation. In other words, they are involved in nervous system degeneration and regeneration (12-14). Other studies showed that CyP-A associates in vitro and in vivo with the dynein/dynactin motor protein complex (including tubulin), which suggests that CyP-A may perform a general function related to the binding of cargo for retrograde movement along microtubules (15). From Fig. 3A, we can see that RAB24 and CyP-A are well colocalized in the perinuclear position, which implied that they may cooperate to function in a signaling pathway at this position or elsewhere. More study results showed that CyP-A plays an essential role in the HIV-1 life cycle, including early and late events in HIV-1 replication (16-18). Moreover, CyP-A has different and even converse effects on HIV-1 infection and replication when expressed in human and monkey cells, i.e., interaction between the capsid protein and host CyP-A protects HIV-1 from restriction in human cells, but is essential for maximal restriction in simian or monkey cells (19-22). Whether or not RAB24 is involved with CyP-A in this process, and the different subcellular distribution of RAB24 is related to the different response to HIV-1 in human and monkey, remains an enigma and needs to be elucidated.

Another interacting protein colocalized with RAB24 (Fig. 3B), GABARAP is a multifunctional protein and could interact with a myriad of binding partners, including the $\gamma^{2}$ subunit of the GABAA receptor, tubulin and microtubules, $\mathrm{N}$-ethyl maleimide sensitive factor (NSF), gephyrin and the transferin receptor (23-29). Surprisingly, RAB24 and GABARAP can colocalize in the cytoplasm (Fig. 3B, upper two panels) or nucleus (Fig. 3B, lower panel). A possible explanation is that some GABARAP could translocate into the nucleus by association with RAB24 in COS-7 cells. It is unknown as to whether this is the case or not. Previous study results showed that RAB24 is colocalized with LC3, a family of yeast Atg8-related proteins (the other two families being GABARAP and GATE16). It has been reported that three families of mammalian Atg8-related proteins were able to localize to the autophagosomal membrane depending on their form-II formation (30). LC3 has been used to study autophagy in neurodegenerative and neuromuscular diseases, tumorigenesis, and bacterial and viral infections.

All results from the previous and present studies have strengthened the hypothesis that RAB24 (with CyP-A and/or GABARAP) is engaged in autophagy and neurodegenerative disorders to some extent, since these two cell processes are confirmed to have a significant relationship (31-37).

Rab24 has several attributes that are different from other members of the Rab family, one being low GTPase activity, which is known from non-human study models such as the mouse. It was shown that this GTPase activity is attributed to human RAB24, which is consistent to previous reports (Fig. 4B). The amino acid substitution at two different positions (Y17A and S67Q) result in a dramatic increase of GTPase activity of RAB24 (Fig. 4C and D), which suggested that these two amino acid sites could contribute to the low GTPase activity of RAB24. Y17, falling within the GXXXVGK (S/T) motif, and S67, falling beside the DXXG motif, appear to be unique compared with other Rab family members (3). From these results, it can be concluded that these two amino acid sites must have negative effects on the GTPase activity of RAB24. 
The mutant RAB24 (D123I) is obviously different from two other mutants since it shifted to form inclusions instead of soluble patterns when expressed in E. coli (Fig. 4D). This phenomenon can also be observed when RAB24 (D123I) is expressed in mammalian cells. Therefore, this mutation site (D123I) is able to induce large changes of conformation of RAB24 and influence the function of this protein, resulting in abnormal cellular responses.

\section{Acknowledgements}

This research was supported by the main project of the National Natural Science Foundation of China (no. 10490193 and 10490190).

\section{References}

1. Olkkonen VM, Dupree P, Killisch I, Lutcke A, Zerial M and Simons K: Molecular cloning and subcellular localization of three GTP-binding proteins of the rab subfamily. J Cell Sci 106: 1249-1261, 1993.

2. Munafo DB and Colombo MI: Induction of autophagy causes dramatic changes in the subcellular distribution of GFP-Rab24. Traffic 3: 472-482, 2002.

3. Erdman RA, Shellenberger KE, Overmeyer JH and Maltese WA: Rab24 is an atypical member of the Rab GTPase family. Deficient GTPase activity, GDP dissociation inhibitor interaction, and prenylation of Rab24 expressed in cultured cells. J Biol Chem 275: 3848-3856, 2000.

4. Maltese WA, Soule G, Gunning W, Calomeni E and Alexander B: Mutant Rab24 GTPase is targeted to nuclear inclusions. BMC Cell Biol 3: 25-41, 2002.

5. He H, Dai F, Yu L, et al: Identification and characterization of nine novel human small GTPases showing variable expressions in liver cancer tissues. Gene Exp 10: 231-242, 2002.

6. Kierczak M, Surmacz L, Wiejak J and Wyroba E: Role of the adaptins, dynamin like GTP-ases and Rab proteins in metabolic disorders and various infections. Postepy Hig Med Dosw 57: 727-737, 2003.

7. Truant $\mathrm{R}$ and Cullen B: The arginine-rich domains present in human immunodeficiency virus type 1 Tat and Rev function as direct importin beta-dependent nuclear localization signals. Mol Cell Biol 19: 1210-1217, 1999.

8. Palmieri D and Malim M: Importin beta can mediate the nuclear import of an arginine-rich nuclear localization signal in the absence of importin alpha. Cell Biol 19: 1218-1225, 1999.

9. Weidenheim KM and Dickson DW: Intranuclear inclusion bodies in an elderly demented woman: a form of intranuclear inclusion body disease. Clin Neuropathol 14: 93-99, 1995.

10. Kleizen B and Braakman I: Protein folding and quality control in the endoplasmic reticulum. Curr Opin Cell Biol 16: 343-349, 2004.

11. Howard BR, Vajdos FF, Li S, Sundquist WI and Hill CP: Structural insights into the catalytic mechanism of cyclophilin A. Nat Struct Biol 10: 475-481, 2003.

12. Avramut $M$ and Achim CL: Immunophilins in nervous system degeneration and regeneration. Curr Top Med Chem 3: 1376-1382, 2003.

13. Gold BG: FK506 and the role of immunophilins in nerve regeneration. Mol Neurobiol 15: 285-306, 1997.

14. Capano M, Virji S and Crompton M: Cyclophilin-A is involved in excitotoxin-induced caspase activate generation. Curr Top Med Chem 3: 1376-1382, 2003.

15. Galigniana MD, Morishima Y, Gallay PA and Pratt WB: Cyclophilin A is bound through its petidylprolyl isomerase domain to the cytoplasmic dynein motor protein complex. J Biol Chem 279: 55754-55759, 2004.

16. Zander K, Sherman MP, Tessmer U, et al: Cyclophilin A interacts with HIV-1 Vpr and is required for its functional expression. J Biol Chem 278: 43202-43213, 2003.
17. Sherry B, Zybarth G, Alfano M, et al: Role of cyclophilin A in the uptake of HIV-1 by macrophages and T lymphocytes. Proc Natl Acad Sci USA 95: 1758-1763, 1998.

18. Braaten D, Franke EK and Luban J: Cyclosporine A-resistant human immunodeficiency virus type 1 mutants demonstrate that Gag encodes the functional target of cyclophilin A. J Virol 70: 3551-3560, 1996.

19. Sayah DM, Sokolskaja E, Berthoux L and Luban J: Cyclophilin A retrotransposition into TRIM5 explains owl monkey resistance to HIV-1. Nature 430: 569-573, 2004.

20. Nisole S, Lynch C, Stoye JP and Yap MW: A Trim5-cyclophilin A fusion protein found in owl monkey kidney cells can restrict HIV-1. Proc Natl Acad Sci USA 101: 13324-13328, 2004.

21. Berthoux L, Sebastian S, Sokolskaja E and Luban J: Lv1 inhibition of human immunodeficiency virus type 1 is counteracted by factors that stimulate synthesis or nuclear translocation of viral cDNA. J Virol 78: 11739-11750, 2004.

22. Ikeda Y, Ylinen LM, Kahar-Bador M and Towers GJ: Influence of gag on human immunodeficiency virus type 1 species-specific tropism. J Virol 78: 11816-11822, 2004.

23. Nymann-Andersen J, Wang H, Chen L, Kittler JT, Moss SJ and Olsen RW: Subunit specificity and interaction domain between GABA(A) receptor-associated protein (GABARAP) and GABA(A) receptors. J Neurochem 80: 815-823, 2002.

24. Coyle JE, Qamar S, Rajashankar KR and Nikolov DB: Structure of GABARAP in two conformations: implications for $\operatorname{GABA}(\mathrm{A})$ receptor localization and tubulin binding. Neuron 33: 63-74, 2002.

25. Wang $\mathrm{H}$ and Olsen $\mathrm{RW}$ : Binding of the GABA(A) receptorassociated protein (GABARAP) to microtubules and microfilaments suggests involvement of the cytoskeleton in GABARAPGABA(A) receptor interaction. J Neuro Chem 75: 644-655, 2000.

26. Wang H, Bedford FK, Brandon NJ, Moss SJ and Olsen RW: GABA(A)-receptor-associated protein links GABA(A) receptors and the cytoskeleton. Nature 397: 69-72, 1999.

27. Kneussel M, Haverkamp S, Fuhrmann JC, Wang H, Wassle H, Olsen RW and Betz H: The gamma-aminobutyric acid type A receptor (GABAAR)-associated protein GABARAP interacts with gephyrin, but is not involved in receptor anchoring at the synapse. Proc Natl Acad Sci USA 97: 8594-8599, 2000.

28. Green F, O'Hare T, Blackwell A and Enns CA: Association of human transferrin receptor with GABARAP. FEBS Lett 518: 101-106, 2002.

29. Coyle JE and Nikolov DB: GABARAP: lessons for synaptogenesis. Neuroscientist 9: 205-216, 2003.

30. Kabeya Y, Mizushima N, Yamamoto A, Oshitani-Okamoto S, Ohsumi Y and Yoshimori T GABARAP and GATE16 localize to autophagosomal membrane depending on form-II formation. J Cell Sci 117: 2805-2812, 2004

31. Klionsky DJ: The molecular machinery of autophagy: unanswered questions. J Cell Sci 118: 7-18, 2005.

32. Marino G and Lopez-Otin C: Autophagy: molecular mechanisms, physiological functions and relevance in human pathology. Cell Mol Life Sci 61: 1439-1454, 2004.

33. Ravikumar B, Vacher C, Berger Z, et al: Inhibition of mTOR induces autophagy and reduces toxicity of polyglutamine expansions in fly and mouse models of Huntington disease. Nat Genet 36: 585-595, 2004.

34. Tanida I, Tanida-Miyake E, Komatsu M, Ueno T and Kominami E: Human Apg3p/Aut1p homologue is 30. An authentic E2 enzyme for multiple substrates, GATE-16, GABARAP, and MAP-LC3, and facilitates the conjugation of hApg12p to hApg5p. J Biol Chem 277: 13739-13744, 2002.

35. Larsen KE and Sulzer D: Autophagy in neurons: a review. Histol Histopathol 17: 897-908, 2002.

36. Jellinger KA and Stadelmann C: Mechanisms of cell death in neurodegenerative disorders. J Neural Transm Suppl 59: 95-114, 2000.

37. Anglade P, Vyas S, Javoy-Agid F, et al: Apoptosis and autophagy in nigral neurons of patients with Parkinson's disease. Histol Histopathol 12: 25-31, 1997. 